\title{
Pressure-Driven DNA in Nanogroove Arrays: Complex Dynamics Leads to Length- and
} Topology-Dependent Separation

\author{
Mikkelsen, Morten Bo Lindholm; Reisner, Walter; Flyvbjerg, Henrik; Kristensen, Anders
}

Published in:

Nano Letters

Link to article, DOI:

$10.1021 / \mathrm{nl} 1044764$

Publication date:

2011

Document Version

Early version, also known as pre-print

Link back to DTU Orbit

Citation (APA):

Mikkelsen, M. B. L., Reisner, W., Flyvbjerg, H., \& Kristensen, A. (2011). Pressure-Driven DNA in Nanogroove Arrays: Complex Dynamics Leads to Length- and Topology-Dependent Separation. Nano Letters, 11(4), 1598-1602. https://doi.org/10.1021/nl1044764

\section{General rights}

Copyright and moral rights for the publications made accessible in the public portal are retained by the authors and/or other copyright owners and it is a condition of accessing publications that users recognise and abide by the legal requirements associated with these rights.

- Users may download and print one copy of any publication from the public portal for the purpose of private study or research.

- You may not further distribute the material or use it for any profit-making activity or commercial gain

- You may freely distribute the URL identifying the publication in the public portal 


\title{
Pressure-Driven DNA in Nanogroove Arrays:
}

\section{Complex Dynamics Leads to Length- and Topology-Dependent Separation}

\author{
Morten Bo Mikkelsen, ${ }^{\dagger}$ Walter Reisner, ${ }^{\dagger}$ Henrik Flyvbjerg, ${ }^{\dagger}$ and Anders \\ Kristensen ${ }^{*} \dagger$
}

DTU Nanotech, Technical University of Denmark, DK-2800 Kgs. Lyngby, Denmark, and

Department of Physics, McGill University, Montreal, Quebec H3A 2T8, Canada

E-mail: anders.kristensen@nanotech.dtu.dk

KEYWORDS: Transport dynamics, Entropic trapping, Confined DNA, Separation

\begin{abstract}
The motion of linear and circular DNA molecules is studied under pressure driven buffer flow in a $50 \mathrm{~nm}$ slit channel with arrays of transverse $150 \mathrm{~nm}$ deep nanogrooves. Transport occurs through two states of propagation unique to this nanogroove geometry: a slow, stepwise groove-to-groove translation called the 'sidewinder' and a fast, continuous tumbling across the grooves, the 'tumbleweed'. Dynamical transitions between the two states are observed at fixed buffer velocity. Molecules exhibit size- and topology-dependent velocities.
\end{abstract}

Nanoconfinement is a powerful tool for controlling polymer conformation and dynamics in lab-on-a-chip type devices for the analysis of DNA and other biomolecules. When a polymer is

\footnotetext{
${ }^{*}$ To whom correspondence should be addressed

${ }^{\dagger}$ DTU Nanotech, Technical University of Denmark, DK-2800 Kgs. Lyngby, Denmark

Department of Physics, McGill University
} 
confined to a space with a dimension below the polymer's gyration radius, the static conformation of the chain will be extended perpendicular to the confinement axis and the chain entropy will be reduced. In sufficiently small nanochannels, e.g. DNA will spontaneously stretch ${ }^{1-4}$, creating an extension along the channel linear with the molecule contour length ${ }^{5}$. Nanochannel devices are thus a convenient platform for performing physical mapping of $\mathrm{DNA}^{6,7}$. In addition, varying confinement in a chip will create global entropy variation and hence free energy gradients that can locally trap DNA, i.e., entropic trapping ${ }^{8,9}$, or drive DNA from confined to open regions of a chip, i.e., entropic recoil ${ }^{10}$.

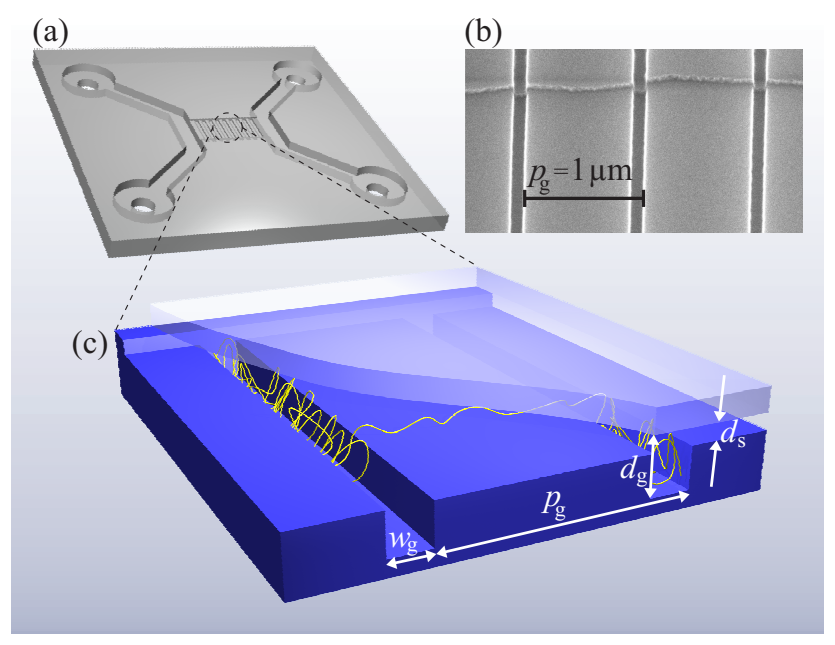

Figure 1: (a) Cartoon of the fluidic device showing inlet reservoirs and microchannels leading to the $450 \mu \mathrm{m}$ long and $50 \mu \mathrm{m}$ wide nanoslit. (b) SEM micrograph showing edge of nanoslit and transverse nanogrooves. (c) Cartoon of nanogroove channel with stretched DNA being transported from one groove to the next. Slit depth $d_{\mathrm{s}}=50 \mathrm{~nm}$, groove depth $d_{\mathrm{g}}=150 \mathrm{~nm}$, groove width $w_{\mathrm{g}}$ is varied from $75 \mathrm{~nm}$ to $600 \mathrm{~nm}$, and periodicity $p_{\mathrm{g}}$ is varied between $1 \mu \mathrm{m}$ and $2.6 \mu \mathrm{m}$.

In this letter we present a device that combines such local manipulation of DNA conformation with entropic trapping ${ }^{8}$. It consists of an array of $150 \mathrm{~nm}$ deep and $75 \mathrm{~nm}$ to $600 \mathrm{~nm}$ wide nanogrooves in the bottom of an open slit, see Figure 1. The depth of the slit $\left(d_{\mathrm{s}}=50 \mathrm{~nm}\right)$ is less than the groove depth $\left(d_{\mathrm{g}}=150 \mathrm{~nm}\right)$, so that in equilibrium a molecule is both extended along a groove and entropically trapped in it. As a molecule is only entropically confined, at sufficient buffer flow, it is driven out of its groove. This leads to a unique form of motion in which an extended molecule is driven transverse to its axis of extension. 
In the classic entropic trapping geometry of Han and Craighead ${ }^{8}$, consisting of traps with lateral dimensions greater than the polymer radius of gyration, the trapped conformation exists in a coil state abutting the slit Figure 2e-f. This coil conformation is approximately that of an unconfined polymer if the field strength in the traps is weak ${ }^{11}$. Larger molecules have a greater probability of escaping the traps than smaller molecules, as larger coils abut a greater slit surface, and hence have a greater probability of forming herniations in the slit that lead to escape ${ }^{8,11}$. This classic geometry has been utilized to perform size separations for DNA in the 1-100 kbp size range for both batch ${ }^{12}$ and continuous flow separation modes ${ }^{13}$.

Our nanogroove geometry, in contrast, alters the confined DNA conformation by extending the molecule in the entropic trap, giving rise to a qualitatively different transport physics. In particular, we observe a new type of self-organized dynamical behavior of a single polymer chain. The hundreds of degrees of freedom of a DNA molecule alternate between a state where an extended DNA molecule can make stochastic hops based on escape of its free ends, which we call the 'sidewinder' (Figure $2 \mathrm{c}-\mathrm{d}$ ), and a compact highly folded state propagating at constant velocity, which we call the 'tumbleweed' (Figure 2b). From a fundamental point-of-view, this system demonstrates that features of complex dynamical behavior ${ }^{14,15}$ can arise in the context of motion of a single polymer chain under a constant driving force in a periodic potential landscape.

While pure motion in either state exhibits a size-independent velocity at fixed buffer velocity, the dynamic interplay between the two states creates a size-dependent velocity that can be utilized for separation. In particular, we show that our device can perform separations based on polymer topology: linear and topologically circular DNA molecules of the same size migrate at different speeds in the nanogroove arrays. This is a striking feature unique to the nanogroove geometry: the classic entropic trapping geometry is in principle much less sensitive to polymer topology. While circular DNA has a slightly more compact conformation in bulk than linear DNA, which should lead to an increased trapping lifetime and a lower migration velocity in the classic geometry, the difference is small; the radii of gyration of ideal linear and circular chains differ by only a factor of $\sqrt{2}^{16}$. 
Molecule dynamics When a solution of DNA is driven through the nanogroove device at buffer velocities below the threshold for escape ${ }^{17}$, a molecule remains extended in the nanogroove while buffer circulates through the slit; see Supporting Information for movie files and experimental conditions. This regime could be used for physical mapping of the extended DNA while performing real-time buffer exchanges. As buffer velocity is slowly increased above the threshold for escape, for linear DNA, end-escape becomes most probable and the molecule will enter the sidewinder state: the escape of a molecule-end prompts a groove-to-groove translation, repeats of which give rise to a motion similar to that of a sidewinder rattlesnake, see Movie2 in Supporting Information. This state of motion is slow and its average velocity is independent of molecule length, because most time is spent waiting for end-escape to occur.

As buffer velocity is still further increased, herniation of contour from the molecule mid-section into the adjacent groove can occur. At low buffer velocity, contour transport is slow and herniation will most likely lead to a sidewinder transition, see Movie4 in Supporting Information. But if the herniated segment that flows into the adjacent groove, flows there too fast to stretch out in the groove as fast as it arrives, the DNA contour in the groove may locally be folded back on itself. Such an increase in local base pair concentration increases self-avoidance interactions, hence decreases the free energy differential between DNA in nanogroove and slit, so that this locally folded DNA is locally less confined. Consequently, there is a high probability of additional herniation of contour from this folded region of the molecule, see Movie 8 in Supporting Information. Thus, at sufficient buffer speed, once they have formed, regions of folded DNA will tend to remain and self-propagate in a characteristic continuously "tumbling" state of motion: the tumbleweed state. Reversions of the tumbleweed to the sidewinder occurs, e.g., if an end-escape occurs and pulls contour out of folded segments before they prompt the next herniation.

Alternations between the two states occur during the dynamics, see Movie1 in Supporting Information, with the tumbleweed becoming dominant as the buffer velocity is increased. Moreover, the longer the molecule, the more probable is herniation as opposed to end-escape ${ }^{18}$, and the more likely it is for the molecule to have folded and compressed regions. As observed, the faster 
tumbleweed state is thus more probable for longer molecules.

Circular DNA can escape via herniation to a circular equivalent of the sidewinder state, see Figure 2d. Propagating circular DNA can also form tumbleweed states when herniated strands in the adjacent nanogroove undergo additional folding, see Movie10 in Supporting Information.

Single molecule analysis Figure 3 shows center-of-mass positions, velocities, and radii of gyration along $\left(R_{\mathrm{g}}^{x}\right)$ and perpendicular $\left(R_{\mathrm{g}}^{y}\right)$ to the grooves for two molecules with different topologies, linear and circular. For both molecules these plots qualitatively demonstrate the existence of the two states of motion, tumbleweed (T) and sidewinder $(\mathrm{S})$ as indicated on the top bar, as well as dynamic transitions between them. The data analysis protocol is described in Sec. 3 in Supporting Information.

The sidewinder state is characterized by single-groove transitions, during which velocity and $R_{\mathrm{g}}^{\mathrm{y}}$ shortly increase while the molecule spans two grooves. $R_{\mathrm{g}}^{\mathrm{x}}$ decreases only slightly during transitions because the molecule is in equilibrium in both grooves. The circular molecule has a greater decrease in $R_{\mathrm{g}}^{\mathrm{x}}$ because of its shorter extension.

The tumbleweed state is characterized by the continuous propagation across multiple grooves of a compressed molecule. Compression is seen as an elevated intensity in the fluorescence images, but it also reduces the molecule's extension along the grooves, resulting in the decrease of $R_{\mathrm{g}}^{x}$ to roughly half the equilibrium extension, while velocity and $R_{\mathrm{g}}^{\mathrm{y}}$ remain high.

Using these criterions to identify sidewinders and tumbleweeds, we can quantitatively demonstrate that they are two distinct states of propagation. The time a molecule is trapped in a single groove, waiting to escape, is referred to as its dwell time $\tau$. The widths of gray areas in Figure 3 are dwell times. Histograms of observed dwell times are shown in Figure 4, Panels a and d, for linear and circular DNA, respectively. Dwell times are random and exponentially distributed, $p(\tau) \propto \exp (-\tau / \bar{\tau})$, for $\tau>0.9 \mathrm{~s}$. This exponential distribution is proof that a molecule, which have dwelled longer than $0.9 \mathrm{~s}$, has constant probability of escape per unit time, independent of how long it has been trapped, i.e., escape is a Poisson process. Such a molecules must be in equi- 
librium with itself in the groove, or its behavior would not be time-invariant. The count of dwell times less than $0.9 \mathrm{~s}$ exceeds the number predicted by the exponential distribution. The excess count could be attributed to molecules that were compressed or folded a little when they landed in the grove. This makes them escape with the higher escape rate that folds and compression give rise to, before they have relaxed to equilibrium. This tumbleweed-like feature could make them tumbleweeds, but with sidewinder features, if we used a different cut-off in our classifier of states. The average velocity of DNA in the sidewinder state is given by $\left\langle v_{\mathrm{S}}\right\rangle=p_{\mathrm{g}} / \bar{\tau}$, where $p_{\mathrm{g}}$ is the distance between neighboring grooves.

Figure 4, Panels $\mathrm{b}$ and e, show histograms of the number $n_{\mathrm{S}}$ of sidewinder transitions made by a molecule between two consecutive periods in the tumbleweed state. Both histograms show clearly that $n_{\mathrm{S}}$ is a random integer with exponential distribution. Thus the transition from the sidewinder to the tumbleweed state is also a Poisson process. Its transition rate is $R_{\mathrm{S} \rightarrow \mathrm{T}}=\left\langle v_{\mathrm{S}}\right\rangle /\left(p_{\mathrm{g}} \bar{n}_{\mathrm{S}}\right)$.

Figure 4, Panels $\mathrm{c}$ and $\mathrm{f}$, show histograms of distances $n_{\mathrm{T}}$ traveled by individual tumbleweeds between two consecutive periods of dwelling as a sidewinder in a nanogroove. The integer $n_{\mathrm{T}}$ measures distance in units of the nanogroove separation $p_{\mathrm{g}}$. It is a random number with exponential probability distribution of values exceeding two groove periods. Thus the transition from the tumbleweed to the sidewinder state is also a Poisson process, showing that the DNA molecule is in a state of dynamical equilibrium with itself when it travels as a tumbleweed. The count of molecules traveling only one or two groove periods greatly exceeds the count suggested by the shown exponential distribution. The excess is due to DNA in the sidewinder state, compressed or folded enough to fool our filter. Some of these molecules make two consecutive groove transitions with so brief a dwelling time between that the molecule registers as a tumbleweed. Molecules traveling longer than two groove periods are bona fide tumbleweeds with a transition rate back to the sidewinder state given by $R_{\mathrm{T} \rightarrow \mathrm{S}}=\left\langle v_{\mathrm{T}}\right\rangle /\left(p_{\mathrm{g}} \bar{n}_{\mathrm{T}}\right)$, where $\left\langle v_{\mathrm{T}}\right\rangle$ is the average velocity of DNA in the tumbleweed state.

The exponentially distributed travel lengths of sidewinders and tumbleweeds show that they are two distinct dynamic states. Both linear $\lambda$-DNA and circular 9-42 charomid DNA molecules 
are seen to randomly alternate between these two states at a given buffer velocity. Many molecules will intermittently be borderline cases. This does not challenge the existence of the two states, but shows that their borderline is not sharp. That does not prevent separation based on the different transport properties of sidewinders and tumbleweeds, see Sec. 5 in Supporting Information, since the average state of motion of a molecule is biased towards one state or the other.

With $P_{\mathrm{S}}$ the probability of being in a sidewinder state and $P_{\mathrm{T}}$ the probability of being in a tumbleweed state, detailed balance gives $P_{\mathrm{T}} R_{\mathrm{T} \rightarrow \mathrm{S}}=P_{\mathrm{S}} R_{\mathrm{S} \rightarrow \mathrm{T}}$ with $P_{\mathrm{S}}+P_{\mathrm{T}}=1$. The molecule velocity is then determined by $V=\left\langle v_{\mathrm{S}}\right\rangle P_{\mathrm{S}}+\left\langle v_{\mathrm{T}}\right\rangle P_{\mathrm{T}}$.

Ensemble analysis Figure 5a shows average velocities as function of buffer velocity for $\lambda$-DNA and T4 GT7-DNA in a slit channel with groove arrays of different width $w_{\mathrm{g}}$. The arrays are placed in series to ensure equal buffer velocities in slit regions.

At low buffer velocity, only sidewinders exist and propagation is very slow. As soon as tumbleweeds form, which happens at a length-dependent threshold, they dominate the dynamics since $\left\langle v_{\mathrm{T}}\right\rangle \gg\left\langle v_{\mathrm{S}}\right\rangle$. This results in the very large velocity ratio $\left\langle v_{\mathrm{T} 4}\right\rangle /\left\langle v_{\lambda}\right\rangle-1$ shown as insert in Figure 5a. In the regime of high buffer velocity, only tumbleweeds exist, and molecules reach a length-independent velocity, experiencing the nanogrooves as an effective friction, see Sec. 4 in Supporting Information. Increasing groove width increases the free energy difference between groove and slit, hence shifts threshold buffer velocities to higher values.

Figure 5b compares average velocities of linear and circular 9-42 charomid DNA molecules. The higher basepair-concentration of circular DNA ${ }^{19}$ reduces the free energy difference between contours in the groove and in the slit. Consequently, the escape probability of circular DNA is higher than that of linear DNA. The velocity ratio $\left\langle v_{\text {circ }}\right\rangle /\left\langle v_{\text {lin }}\right\rangle=8$ at $v_{\mathrm{s}}=35 \mu \mathrm{m} / \mathrm{s}$ shows that the system can be used for separation of linear and circular molecules; see Movie 9 in Supporting Information. This difference is much greater than would be expected for the classic entropic trapping geometry. In the classic geometry, we would expect the mobility $\mu$ of linear and circular molecules to differ by no more than $30 \%$, with the bound arising from the ratio of the radii of gyration of 
ideal linear and circular chains $\left(\mu_{\text {circ }} / \mu_{\text {lin }}>R_{\mathrm{g}}^{\text {circ }} / R_{\mathrm{g}}^{\operatorname{lin}} \sim 1 / \sqrt{2} \approx 0.7\right)$.

Conclusion We have found and characterized two distinct states of motion, 'sidewinder' and 'tumbleweed', which are observed for both linear and circular DNA molecules in nanogroove arrays. The characteristic features of these states emerge as the many degrees of freedom of the polymer interact repeatedly with the nanotopology. The demonstrated device can be used for molecule filtering, DNA separation, and sample preparation. In particular, it possesses the unique property that motion of DNA in the arrays is sensitive to molecule topology as well as size.

\section{Acknowledgement}

The work was partly funded by the Danish Council for Technology and Innovation through the Innovation Consortium OCTOPUS, by the Danish Research Council for Technology and Production, FTP grant no. 274-05-0375, and by the European Commission funded project READNA (Contract HEALTH-F4-2008-201418).

Supporting Information Available: Movie files and details on experiments and data analysis. This material is available free of charge via the Internet at http://pubs.acs.org.

\section{References}

(1) W. Reisner, K. J. Morton, R. Riehn, Y. M. Wang, Z. Yu., M. Rosen, J. C. Sturm, S. Y. Chu, E. Frey, R. H. Austin, Phys. Rev. Lett. 94, 196101 (2005)

(2) W. Reisner, J. Beech, N. Larsen, H. Flyvbjerg, A. Kristensen, J.O. Tegenfeldt, Phys. Rev. Lett. 99, 058302 (2007)

(3) K. Jo, D. Dhingra, T. Odijk, J. de Pablo, M. Graham, R. Runnheim, D. Forrest, D. Schwartz, Proc. Natl. Acad. Sci. USA 104, 2673 (2007)

(4) M. Krishnan, I. Monch, P. Schwille, Nano Lett. 7, 1270 (2007) 
(5) J. O. Tegenfeldt, C. Prinz, H. Cao, S. Chou, W. Reisner, R. Riehn, Y. M. Wang, E. C. Cox, J. C. Sturm, P. Silberzan., R. H. Austin, Proc. Natl. Acad. Sci. USA 101, 10979 (2004)

(6) R. Riehn, M. Lu, Y. M. Wang, S. F. Lim, E. C. Cox, R. H. Austin, Proc. Natl. Acad. Sci. USA 102, $10012(2005)$

(7) Y. M. Wang, J. O. Tegenfeldt, W. Reisner, R. Riehn, X. J. Guan, L. Guo, I. Golding, E. C. Cox, J. Sturm, R. H. Austin, Proc. Natl. Acad. Sci USA 102, 9796 (2005)

(8) J. Han, S.W. Turner, H.G. Craighead, Phys. Rev. Lett. 83, 1688 (1999)

(9) J. Han, H.G. Craighead, Science 288, 1026 (2000)

(10) S.W. Turner, M. Cabodi, H.G. Craighead, Phys. Rev. Lett. 88, 128103 (2002)

(11) J. Han, H. Craighead, Anal. Chem. 74, 395 (2002)

(12) J. Fu, J. Yoo, J. Han, Phys. Rev. Lett. 97, 018103 (2006)

(13) J. Fu, R. B. Schoch, A. L. Stevens, S. R. Tannenbaum, J. Han, Nature Nano 2, 121 (2007)

(14) V. Schaller, C. Weber, C. Semmrich, E. Frey, A. R. Baush, Nature 467 73-77 (2010)

(15) H. Haken: Synergetics, an Introduction: Nonequilibrium Phase Transitions and SelfOrganization in Physics, Chemistry, and Biology, 3rd rev. enl. ed., Springer-Verlag, New York, 1983.

(16) M. Rubinstein, R. H. Colby, Polymer Physics (Oxford University Press, New York, 2003)

(17) T. Sakaue, Eur. Phys. J. E. 19, 477-487 (2006)

(18) K. L. Sebastian and Alok K. R. Paul, Phys. Rev. E 62, 927-939 (2000)

(19) F. Persson, P. Utko, W. Reisner, N. B. Larsen, A. Kristensen, Nano Letters 9, 1382-1385 (2009) 


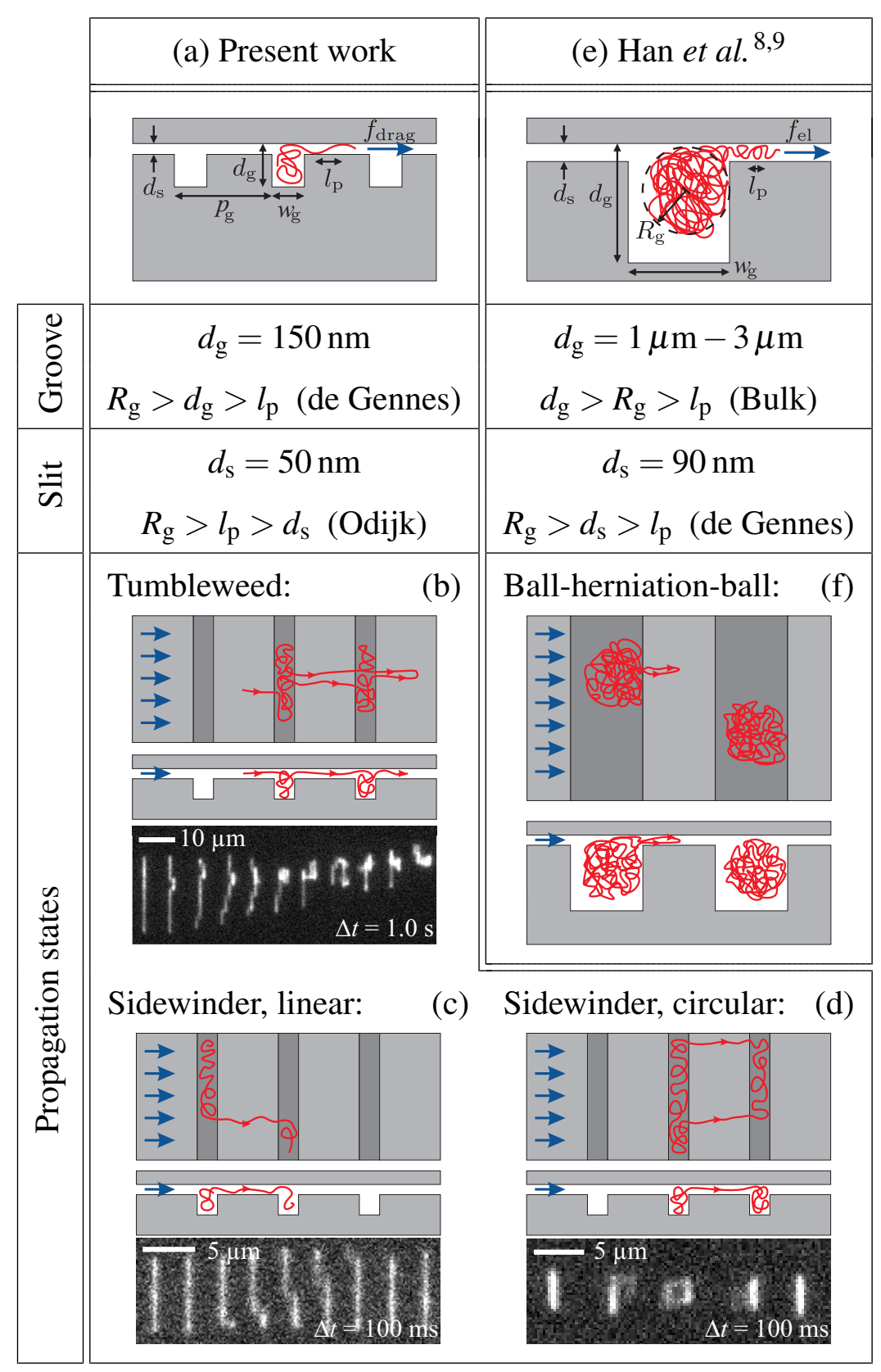

Figure 2: Propagation states of DNA in various geometries. Arrowheads on DNA (red lines) indicate direction of motion of DNA that flows in response to buffer flow shown as blue arrows. (a) Alternating de Gennes and Odijk confinement regimes ${ }^{1}$ stretch DNA in front of the slit, which gives rise to two distinct propagation states: the folded 'tumbleweed' state (b), and the stretched 'sidewinder' state in case of (c) linear DNA and (d) circular DNA. This contrasts with the single propagation state (f) seen in Han and Craighead's device (e), in which alternating bulk and de Gennes regimes allow DNA to coil in a ball conformation in front of the slit, and transport through the slit is initiated by herniation from the ball. $l_{\mathrm{p}}$ is persistence length and $R_{\mathrm{g}}$ gyration radius. 


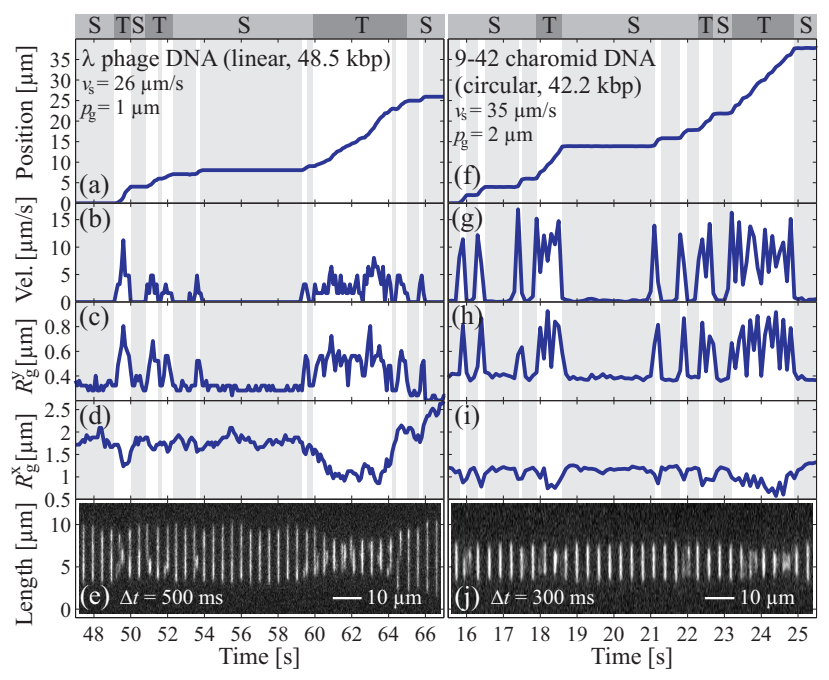

Figure 3: Data extracted from movies of linear $\lambda$-DNA (a-e) and circular 9-42 charomid DNA (f$\mathrm{j}$ ) in slit channels with groove period $p_{\mathrm{g}}$ and buffer velocity $v_{\mathrm{s}}$. Gray background indicate trapping of molecule and confinement to a single groove. Bar on top indicates the state of the molecules, tumbleweed (T) or sidewinder (S). Graphs have time resolution $\Delta t=100 \mathrm{~ms}$ and show the molecules' center-of-mass positions, velocities, and radii of gyration along $\left(R_{\mathrm{g}}^{x}\right)$ and perpendicular $\left(R_{\mathrm{g}}^{y}\right)$ to the grooves. $(e, j)$ Fluorescence image montages corresponding to graphs.
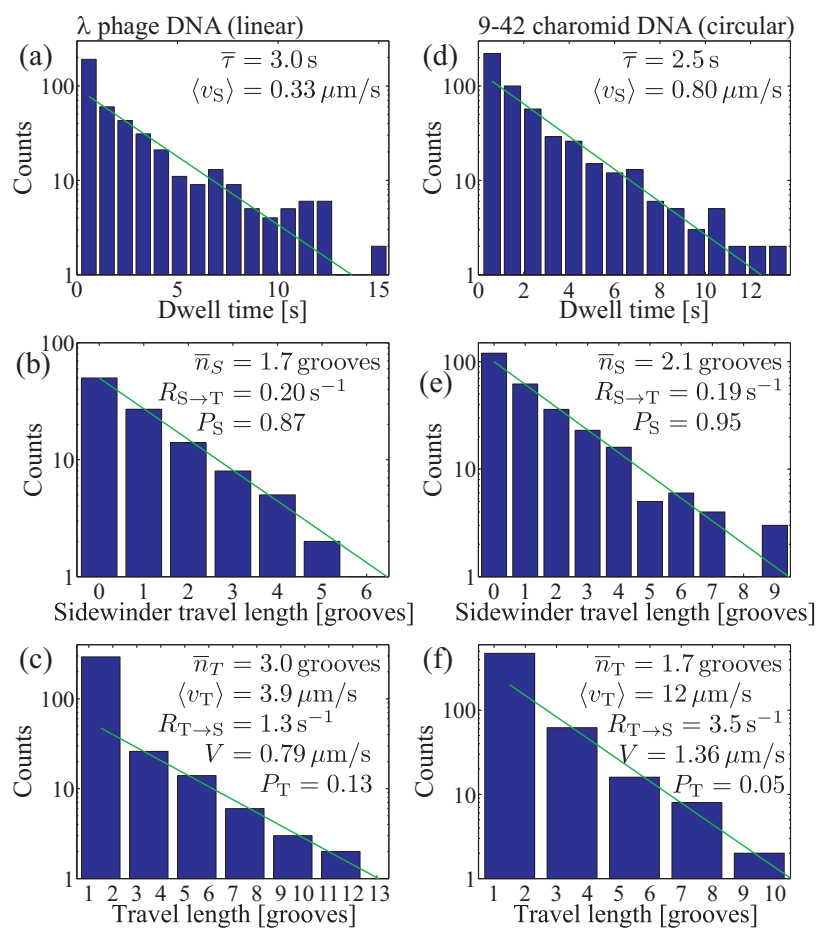

Figure 4: Histograms of dwell time $\tau$, sidewinder travel length $n_{\mathrm{S}}$, and tumbleweed travel length $n_{\mathrm{T}}$. (a-c) $\lambda$-DNA with $p_{\mathrm{g}}=1 \mu \mathrm{m}$ and $v_{\mathrm{s}}=26 \mu \mathrm{m} / \mathrm{s}$. (d-f) Circular 9-42 charomid DNA with $p_{\mathrm{g}}=2 \mu \mathrm{m}$ and $v_{\mathrm{s}}=35 \mu \mathrm{m} / \mathrm{s}$. Characteristic times, transition rates, and probabilities given in panels characterize the exponential fits shown. Calculated velocities $V$ correspond well to the measured values $\left\langle v_{\text {lin }}\right\rangle=0.87 \mu \mathrm{m} / \mathrm{s}$ and $\left\langle v_{\text {circ }}\right\rangle=1.66 \mu \mathrm{m} / \mathrm{s}$. 

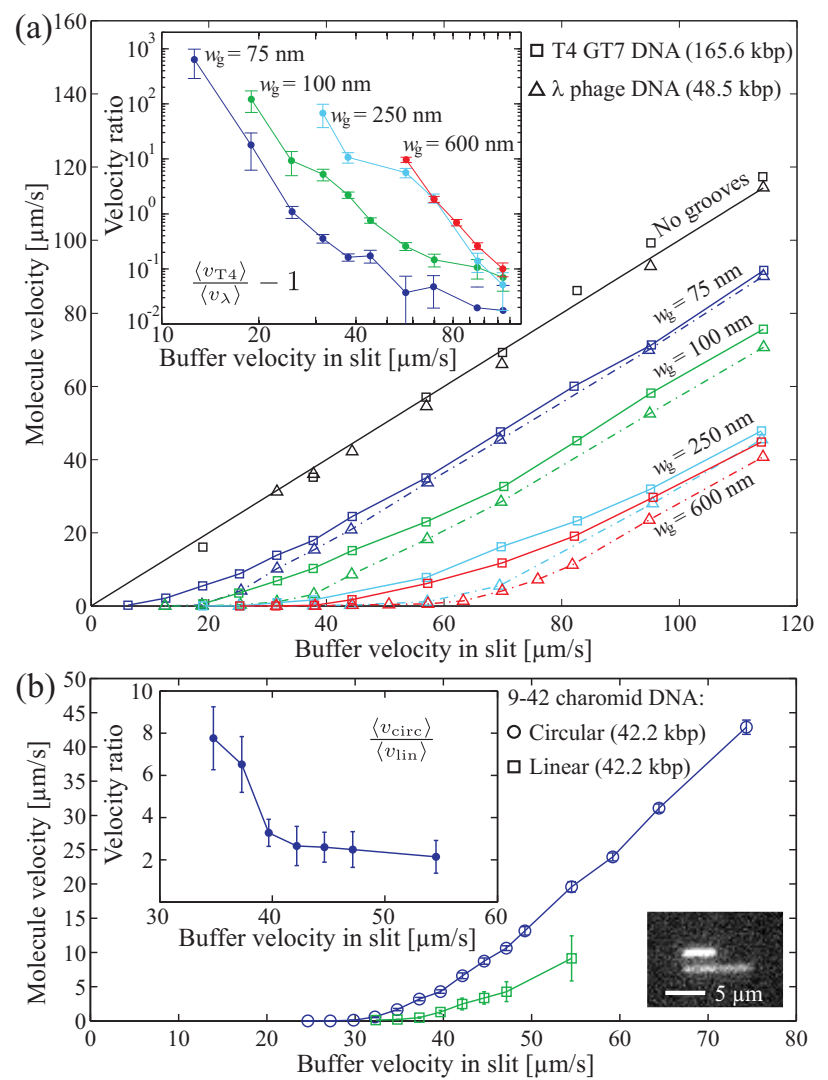

Figure 5: (a) Molecule velocity as function of buffer velocity in groove arrays of different width $w_{\mathrm{g}}$. Groove period $p_{\mathrm{g}}=w_{\mathrm{g}}+2 \mu \mathrm{m}$. Insert shows velocity ratio between T4 GT7-DNA and $\lambda$ DNA $\left\langle v_{\mathrm{T} 4}\right\rangle /\left\langle v_{\lambda}\right\rangle-1$ in a double-logarithmic plot. Velocity ratios of the order of 100 are seen for $75 \mathrm{~nm}$ grooves. Data based on movies of $1702 \lambda$-DNA and 1460 T4 GT7-DNA molecules. Buffer velocity measured as the average molecule velocity in a groove-free region of the slit channel. (b) Molecule velocity as function of buffer velocity for circular (intact) and linear (broken once) 9-42 charomid DNA. Fluorescence image shows circular DNA (top) and linear DNA (bottom) trapped in adjacent grooves. $w_{\mathrm{g}}=100 \mathrm{~nm}$ and $p_{\mathrm{g}}=2 \mu \mathrm{m}$. Inserted graph shows velocity ratio of circular and linear molecules $\left\langle v_{\text {circ }}\right\rangle /\left\langle v_{\text {lin }}\right\rangle$. Data based on movies of 479 circular and 69 linear 9-42 charomid DNA molecules. 